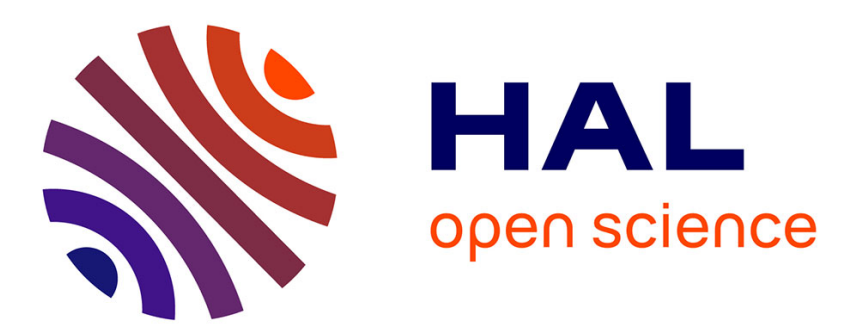

\title{
Ergodicity of conservative communication networks
}

\author{
Dmitri D. Botvich, A.A. Zamyatin
}

\section{To cite this version:}

Dmitri D. Botvich, A.A. Zamyatin. Ergodicity of conservative communication networks. [Research

Report] RR-1772, INRIA. 1992. inria-00077012

\section{HAL Id: inria-00077012 \\ https://hal.inria.fr/inria-00077012}

Submitted on 29 May 2006

HAL is a multi-disciplinary open access archive for the deposit and dissemination of scientific research documents, whether they are published or not. The documents may come from teaching and research institutions in France or abroad, or from public or private research centers.
L'archive ouverte pluridisciplinaire HAL, est destinée au dépôt et à la diffusion de documents scientifiques de niveau recherche, publiés ou non, émanant des établissements d'enseignement et de recherche français ou étrangers, des laboratoires publics ou privés. 
UNITÉ DE RECHERCHE INRIA-ROCQUENCOURT

\section{Rapports de Recherche}

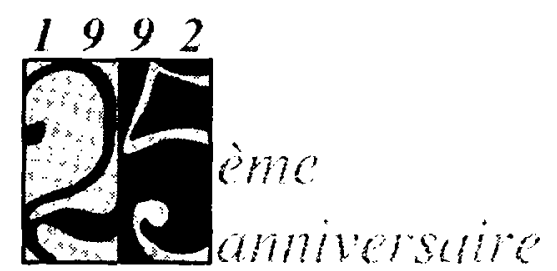

$N^{\circ} 1772$

\section{Programme 1}

Architectures paralleles, Bases de données,

Réseaux et Systèmes distribués

\section{ERGODICITY OF CONSERVATIVE COMMUNICATION NETWORKS}

Institut National de Recherche

en Informatique et en Automatique

Domaine de Voluceau

Rocquencourt

$$
\text { B.P.105 }
$$

78153 Le Chesnay Cedex

France

Dmitri D. BOTVICH

Andrei A. ZAMYATIN

Octobre 1992

Tél:(1)39635511 


\title{
Ergodicity of Conservative Communication Networks
}

\section{Ergodicité de Réseaux de Communications Conservatifs}

\author{
Dmitri D.Botvich * Andrei A.Zamyatin ${ }^{\dagger}$ \\ 9 octobre 1992
}

This paper starts a series of publications entering into the framework of the collaboration between the project MEVAL and the LLRS, as specified in the agreement between INRIA and MGU (Moscow State University).

\section{Editors: Guy Fayolle and Vadim Malyshev.}

\begin{abstract}
We analyze a communication network with several types of calls. For a wide class of conservative service disciplines, we give ergodicity criteria. Exponentially fast convergence to steady state is also proved.

\section{Résumé}

Nous analysons un réseau de télécommunications avec plusieurs types d'appels. Pour une classe asez large de disciplines conservatives, nous donnons des critères d'ergodicité et prouvons, en passant, la convergence exponentielle vers l'état stationnaire.
\end{abstract}

- Postal address: Telecommunications, School of Electronic Engeneering, Dublin City University, Glasnevin, Dublin 9, Ireland. On leave of absence from the LLRS (Laboratory of Large Random Systems), Faculty of Mechanics and Mathematics, Moscow State University.

${ }^{\dagger}$ Postal address: Laboratory of Problems of Theoretical Cybernetics, Faculty of Mechanics and Mathematics, Moscow State University, 119899, Moscow, Russia. 


\section{Introduction.}

We shall study ergodic properties of a communication network with several types of calls when the service time depends on a type of the call. Usually ergodic conditions for communication networks are deduced from explicit formulae for stationary probabilities. For example, in case of Jackson network sufficient ergodicity conditions follow from the product form stationary probabilities. But in many cases equilibrium state probabilities have no product form. Moreover, formulae for stationary probabilities are often impossible to use effectively for obtaining ergodic conditions. For example, a general random walk in $Z_{+}^{2}$ represents a communication network with two servers. But it is known [1], that its stationary probabilities can be expressed only in terms of elliptic integrals.

Explicit expressions for stationary probabilities are impossible to obtain for communication networks with several types of calls when the service time of a call depends on its type. To study ergodicity we shall use Lyapounov function method, which was applied to communication networks in [3 - 6]. In this paper we prove ergodicity criteria for some communication networks which have no explicit formulae. More exactly, we consider a network which is an example of Kelly network [2]. It consists of two nodes. Suppose that there are two types of calls. The calls of type $k$ arrive at the node $k$ in the Poisson stream of rate $\lambda_{k}$. The calls of the first type follow the route $\{1,2\}$, i.e. after completing service in the node 1 the calls of this type enter the node 2. The calls of the second type follow the route $\{2,1\}$. A call of type $k$ at node $i$ requires an amount of service time exponentially distributed with parameter $\mu_{i}(k)$.

We analyse ergodic properties of the above network for a wide class of conservative service disciplines (see below). It means that these disciplines do not allow a server to be empty if the queue is non-empty. The conservative service disciplines appear in communication networks in which it is difficult or unprofitable to support some specific discipline. One of our aims is to prove an ergodicity criterion uniformly over all conservative service disciplines. Let us now introduce the loads

$$
\begin{aligned}
& \rho_{1}(1)=\lambda_{1} / \mu_{1}(1), \rho_{1}(2)=\lambda_{2} / \mu_{1}(2), \\
& \rho_{2}(1)=\lambda_{1} / \mu_{2}(1), \rho_{2}(2)=\lambda_{2} / \mu_{2}(2),
\end{aligned}
$$




$$
\rho_{1}=\rho_{1}(1)+\rho_{1}(2), \rho_{2}=\rho_{2}(1)+\rho_{2}(2),
$$

where $\rho_{i}(k)$ is the load of type $k$ at the $i$-th node and $\rho_{i}$ is the total load over all call types at the $i$-th node.

It turns out, if the following inequalities are fulfilled

$$
\rho_{1}<1, \rho_{2}<1, \rho_{1}(2)+\rho_{2}(1)<1,
$$

then the communication network is ergodic under any conservative service discipline. We also prove that the Markov chain which describes the network has exponential convergence property. It means that the time $t$ transition probabilities exponentially fast converge to the stationary probabilities as $t \rightarrow \infty$ ( Theorem 1 ).

If conditions (1.2) are not fulfilled, i.e. the left part of one of the inequalities is strictly greater than 1 , one can give an example of the conservative service discipline with absolute priorities when the network is not ergodic (Theorem 2). It was observed in [7] that the standard ergodicity conditions $\rho_{1}<1, \rho_{2}<1$ are not sufficient in this case. But for this case an ergodicity criterion was not obtained in [7].

We also consider other disciplines with absolute priorities and show that the conditions $\rho_{1}<1, \rho_{2}<1$ are sufficient for ergodicity (Theorem 3 ).

It was proved in [7] that the standard ergodicity conditions are sufficient for the network with FCFS (first come - first serve) discipline at both nodes.

\section{Main Definitions and Results.}

Let us define a class of conservative service disciplines. We suppose that the complete information about the queues in the network is available. For every call in the network the following characteristics are assumed to be known: a position in queue, a type, a total service time, a residual service time. The state of the $i$-th node is represented by the vector

$$
q=\left(n_{j}, t_{j}, s_{j}, j=1, \ldots, x_{i}\right),
$$

where $n_{j}$ is the type of the $j$-th call in the queue, $t_{j}$ is the residual service time, $s_{j}$ is the total service time and $x_{i}$ is the queue size in the $i$-th node. The difference $s_{j}-t_{j}$ is the received service time, i.e. an amount of service time which the $j$-th call has been received. The collection

$$
\bar{q}=\left(q_{i}, i=1,2\right)
$$


gives the complete description of the queues in the network. The disciplines under study will depend on these data. We suppose that only one call can be served at every moment and the call being served is in the first position $(j=1)$. After arrival at a server a call can remain in the server for some period which we shall call the call sojourn time. If the call has not been served for this period then it leaves the server but remains in the queue. It is supposed that losses of calls are impossible. Call sojourn times are assumed to be exponentially distributed random variables. These random variables are independent of call service times and received service times.. If call sojourn time is infinite then parameters of respective exponential distributions are assumed to be 0 .

Consider moments when a call arrives at some queue, leaves some node or the sojourn time of a current call runs out. We shall call such moments the events. Describe possible changes in nodes. when the events are occured. If a new call arrives then the queue size increases by 1 . If a call sojourn time runs out then queue size is not changed. If a call leaves a queue then the queue size decreases by 1 . Besides that, rearrangements of calls in the queues can take place with conservation of residual service times, total service times and types. These rearrangements are arbitrary functions of $\bar{q}$, where $\bar{q}$ is defined by (2.1). Observe that an interruption of a call service can take place when the events are occured. The above class of disciplines we shall denote by $\mathcal{D}$, but a discipline in the $i$-th node - by $D^{(i)}$. Then the collection

$$
D=\left(D^{(1)}, D^{(2)}\right) \in \mathcal{D} \times \mathcal{D}
$$

defines the service discipline in the whole network.

Let us note that disciplines with absolute priorities are included in the above class. We define now all possible disciplines with absolute priorities for the network under study:

$D_{1}(p)$ - when calls of the first type have absolute priority in both nodes;

$D_{2}(p)$ - when calls of the second type have absolute priority in both nodes;

$D_{3}(p)$ - when calls of the first type have absolute priority in the first node and calls of the second type have absolute priority in the second node;

$D_{4}(p)$ - when calls of the first type have absolute priority in the second node and calls of the second type have absolute priority in the first node;

Let us fix some discipline $D \in \mathcal{D} \times \mathcal{D}$. We have supposed that all service times have exponential distribution and calls arrive in the Poisson stream. 
Then the network is represented by a continuous time Markov chain $\eta_{t}$ with the state space $\mathcal{A}_{1} \times \mathcal{A}_{2}$, where $\mathcal{A}_{i}$ is the set of vectors $\alpha_{i}=\left\{n_{1}, \ldots, n_{x_{i}}\right\}$, and $n_{j}$ is the type of the $j$-th call in the $i$-th queue, $x_{i}$ is the queue size in the $i$-th node.

Let us consider all possible transitions in the network: an arrival of a call of the first (the second) type at the first (the second) node, a departure of a call of the first (the second) type from the network, a departure of a call of the first (the second) type from the first (the second) node and its arrival at the second (the first) node. For example, let a call of the first type leave the first node and arrive at the second node. Then the length of the vector $\alpha_{1}$ decreases by 1 and a rearrangment of calls in the first queue is occured in accordance with a given service discipline. The length of the vector $\alpha_{2}$ increases by 1 and a new vector $\alpha_{2}$ is defined in accordance with a given service discipline. For example, if we use LIFO (last in - first out) discipline then a new call will be placed in the first position. Besides that, rearrangements of calls are possible when the sojourn time of a current call in a server runs out but these transitions do not change queue sizes.

The chain $\eta_{t}$ is defined by transition rates $\lambda_{\alpha \beta}$, where $\alpha, \beta \in \mathcal{A}_{1} \times \mathcal{A}_{2}$. Let us define a discrete time Markov chain $\eta_{n}$ with the transition probabilities

$$
p_{\alpha \beta}=w \lambda_{\alpha \beta}, \alpha \neq \beta, p_{\alpha \alpha}=1-\sum_{\alpha \neq \beta} p_{\alpha \beta}
$$

where a constant $w$ satisfies the condition

$$
w \leq \min _{\alpha}\left(\sum_{\beta} \lambda_{\alpha \beta}\right)^{-1} .
$$

The continuous chain $\eta_{t}$ is ergodic iff the discrete chain $\eta_{t}$ is ergodic and their stationary distributions coincide. Let $\pi(\beta), \beta \in \mathcal{A}_{1} \times \mathcal{A}_{2}$, be the stationary distribution and $\|\beta\|$ be the total number of calls in the network being in the state $\beta$. Let us denote by $\hat{p}_{\alpha \beta}^{(t)}$ the time $t$ transition probabilities for Markov chain $\eta_{t}$.

Now we formulate the main results.

\section{Theorem 1 If}

$$
\rho_{1}<1, \rho_{2}<1, \rho_{1}(2)+\rho_{2}(1)<1
$$


then the network is ergodic for any discipline $D \in \mathcal{D} \times \mathcal{D}$. Moreover, the following estimates are fulfilled:

1) the exponential estimate for the stationary probabilities:

$$
\pi(\beta) \leq C \exp (-\gamma\|\beta\|)
$$

for some constants $C, \gamma>0$ not depending on $\beta$;

2) exponential convergence to the stationary probabilities: there exists a constant $\sigma>0$ such that for all $\alpha, \beta \in \mathcal{A}_{1} \times \mathcal{A}_{\epsilon}$ and $t>0$

$$
\left|\pi(\beta)-\hat{p}_{\alpha \beta}^{(t)}\right| \leq C_{1}(\alpha) \exp (-\sigma t)
$$

for some constant $C_{1}(\alpha)$ depending only on $\alpha$.

Theorem 2 If

$$
\rho_{1}<1, \rho_{2}<1, \rho_{1}(2)+\rho_{2}(1)>1,
$$

or $\rho_{i}>1$ for some $i=1,2$, then the network with the service discipline $D_{4}(p)$ is transient.

Remark. The case when

$$
\rho_{1}<1, \rho_{2}<1, \rho_{1}(2)+\rho_{2}(1)=1
$$

is a special one requiring additional analysis.

For the disciplines $D_{1}(p), D_{2}(p), D_{3}(p)$ we have another situation.

Theorem 3 The network with the disciplines $D_{1}(p), D_{2}(p), D_{3}(p)$ is ergodic if

$$
\rho_{1}<1, \rho_{2}<1
$$

and the estimates (2.2), (2.3) are fulfilled.

Remark. For the disciplines $D_{1}(p), D_{2}(p)$ the first part of this result (ergodicity) has been proved in a more general case [10]. 


\section{Proof of Theorem 1.}

Further we shall use the following functions of $\eta_{n}$. Let $\xi_{n, i}(k)$ be the number of calls of type $k$ in the $i$-th node at time $n$ and $\kappa_{n, i}$ be the type of the call being served at time $n$. Define the random sequences

$$
\begin{gathered}
\xi_{n}=\left(\xi_{n, i}(k), i, k=1,2\right) \in Z_{+}^{4}, \\
\kappa_{n}=\left(\kappa_{n, i}, i=1,2\right) \in\{1,2\} \times\{1,2\},
\end{gathered}
$$

and put

$$
\xi_{n, i}=\xi_{n, i}(1)+\xi_{n, i}(2) .
$$

Note, that $\xi_{n, i}$ is the length of the $i$-th queue at time $n$. Let $x_{i}(k)$ be the values of random variables $\xi_{n, i}(k)$ and $x_{i}$ be the values of random varibles $\xi_{n, i}$. Define the vector

$$
x=\left(x_{i}(k), i, k=1,2\right) \in Z_{+}^{4}
$$

and the orthogonal basis

$$
\left\{e_{i}(k), i, k=1,2\right\}
$$

such that

$$
x=\sum_{k} \sum_{i} x_{i}(k) e_{i}(k)
$$

We shall construct a Laypounov function $G(x)$ depending only on a vector $x$ such that for some $\epsilon>0$ and $m \in Z_{+}$

$$
E\left(G\left(\xi_{n}\right) \mid \xi_{1}=x\right) \leq G(x)-\epsilon
$$

for all $x \in Z_{+}^{4} \backslash B$, where $B$ is a finite set. Then ergodicity will follow from Foster criterion [8]. Moreover, following [3] we give two constructions of Laypounov functions.

First construction. Let us first define linear functions of $x$ :

$$
\begin{gathered}
A_{1,1}(x)=\frac{x_{1}(1)}{\mu_{1}(1)}, \\
A_{1,2}(x)=\frac{x_{1}(2)+x_{2}(2)}{\mu_{1}(2)},
\end{gathered}
$$




$$
\begin{gathered}
A_{2,1}(x)=\frac{x_{2}(1)+x_{1}(1)}{\mu_{2}(1)}, \\
A_{2,2}(x)=\frac{x_{2}(2)}{\mu_{2}(2)}
\end{gathered}
$$

If the network is in the state $x$ then the server at the $i$-th node needs at an average the time $A_{i, k}(x)$ for servicing all calls of type $k$. Define also the linear functions:

$$
A_{i}(x)=A_{i, 1}(x)+A_{i, 2}(x)
$$

and

$$
\begin{aligned}
G_{1}(x) & =\frac{\left(1-\rho_{1}(2)\right) A_{1,1}(x)+\left(1+\delta_{1}\right) \rho_{1}(1) A_{1,2}(x)}{\rho_{1}(1)} \\
G_{2}(x) & =\frac{\left(1-\rho_{2}(1)\right) A_{2,2}(x)+\left(1+\delta_{2}\right) \rho_{2}(2) A_{2,1}(x)}{\rho_{2}(2)}
\end{aligned}
$$

where $\delta_{i}>0, i=1,2$, are small parameters which are chosen in the speciall manner.

To construct a Lyapounov function we need the following lemmas.

Lemma 3.1 If $\rho_{1}<1, \rho_{2}<1$ then there exist parameters $\epsilon, \delta_{1}, \delta_{2}>0$ such that

$$
E\left(G_{i}\left(\xi_{n+1}\right)-G_{i}\left(\xi_{n}\right) \mid \xi_{n, i}>0\right)<-\epsilon .
$$

Proof. Let $i=1$ and a call of the first type be in progress at a given moment, i.e. $\kappa_{n, 1}=1$. Then the one-step mean increment of the function $G\left(\xi_{n}\right)$ is

$$
\begin{gathered}
E\left(G_{1}\left(\xi_{n+1}\right)-G_{1}\left(\xi_{n}\right) \mid \xi_{n, i}>0\right)= \\
=\left(1-\rho_{1}(2)\right) \rho_{1}(1)-\left(1-\rho_{1}(2)\right)+\left(1+\delta_{1}\right) \rho_{1}(1) \rho_{1}(2)= \\
=\rho_{1}-1+\delta_{1} \rho_{1}(1) \rho_{1}(2)<0
\end{gathered}
$$

for sufficiently small $\delta_{1}$. If a call of the second type is in progress at a given moment, i.e. $k_{n, 1}=2$, then

$$
\begin{gathered}
E\left(G_{1}\left(\xi_{n+1}\right)-G_{1}\left(\xi_{n}\right) \mid \xi_{n, i}>0\right)= \\
\left.=\left(1-\rho_{1}(2)\right) \rho_{1}(1)-\left(1+\delta_{2}\right) \rho_{1}(1)\right)+\left(1+\delta_{2}\right) \rho_{1}(1) \rho_{1}(2)= \\
=-\delta_{1} \rho_{1}(1)+\delta_{1} \rho_{1}(1) \rho_{1}(2)<0
\end{gathered}
$$


for all $\delta_{1}>0$.

In the case $i=2$ analogous inequalities are fulfilled.

Define now the two-dimensional faces

$$
\begin{aligned}
& \Lambda_{1}^{(2)}=\left\{x_{2}(k)=0, x_{1}(k)>0\right\}, \\
& \Lambda_{2}^{(2)}=\left\{x_{1}(k)=0, x_{2}(k)>0\right\} .
\end{aligned}
$$

If $x \in \Lambda_{1}^{(2)}$ the second node is empty; if $x \in \Lambda_{2}^{(2)}$ the first node is empty.

Lemma 3.2 If the condition (1.2) is fulfilled then there exist $\delta_{i}>0, i=1,2$, such that the inequality (3.5) holds and for $x \in \Lambda_{1}^{(2)}$ takes place

$$
G_{2}(x)<G_{1}(x)
$$

but for $x \in \Lambda_{2}^{(2)}$ does

$$
G_{2}(x)>G_{1}(x) .
$$

Proof. Let $x \in \Lambda_{1}^{(2)}$. Then

$$
\begin{gathered}
G_{2}(x)=\frac{\left(1+\delta_{2}\right) x_{1}(1)}{\mu_{2}(1)} \\
G_{1}(x)=\frac{\left(1-\rho_{1}(2)\right) x_{1}(1)}{\lambda_{1}}+\frac{\left(1+\delta_{1}\right) x_{1}(2)}{\mu_{1}(2)} .
\end{gathered}
$$

The inequality

$$
\frac{1+\delta_{2}}{\mu_{2}(1)}<\frac{1-\rho_{1}(2)}{\lambda_{1}}
$$

is fulfilled as it is equivalent to the inequality

$$
\rho_{1}(2)+\rho_{2}(1)+\delta_{2} \rho_{2}(1)<1,
$$

which holds for sufficiently small $\delta_{2}$. The case $x \in \Lambda_{2}^{(2)}$ is considered in the same way.

Let us define the Lyapounov function

$$
G(x)=\max \left(G_{1}(x), G_{2}(x)\right),
$$

where the functions $G_{1}(x), G_{2}(x)$ are defined by (3.4).

Suppose that a point $x \in Z_{+}^{4}$ is sufficiently far from faces $\Lambda_{1}^{(2)}, \Lambda_{2}^{(2)}$ i.e. queue sizes $x_{i}$ are large in both nodes. 
Lemma 3.3 There exist $\epsilon, r>0, m \in Z_{+}$such that

$$
E\left(G\left(\xi_{m}\right) \mid \xi_{1}=x\right) \leq G(x)-\epsilon
$$

for all $x$ satisfying the condition

$$
\min \left(x_{1}, x_{2}\right)>r
$$

Proof. The proof of this lemma is analogous to the proof of lemma 5.3 from [3]. In accordance with (3.5) the random sequence

$$
G_{i}\left(\xi_{1}\right), G_{i}\left(\xi_{2}\right), \ldots, G_{i}\left(\xi_{n}\right)
$$

is a supermartingale. Moreover, it has the property

$$
E\left(G_{i}\left(\xi_{n+1}\right) \mid G_{i}\left(\xi_{n}\right), \ldots, G_{i}\left(\xi_{1}\right)\right)<G_{i}\left(\xi_{n}\right)-\sigma
$$

for some $\sigma>0$. By lemma $1.1[\delta]$ the exponential estimate

$$
P\left(G_{i}\left(\xi_{m}\right)-G_{i}\left(\xi_{1}\right)<-\epsilon_{i} m\right)>1-C_{i} e^{-\delta_{i} m},
$$

holds for some $C_{i}, \delta_{i}, \epsilon_{i}>0$. Hence, choosing sufficiently large $\mathrm{r}$ we get that for all i simultaneously

$$
P\left(G_{i}\left(\xi_{m}\right)-G_{i}\left(\xi_{1}\right)<-\epsilon m\right)>1-C e^{-\delta m},
$$

for some constants $C, \delta, \epsilon>0$. The result of the lemma follows easily from this estimate.

Let now a point $x$ be close by one of the two-dimensional faces $\Lambda_{i}^{(2)}$. The following lemma is true.

Lemma 3.4 There exist $\epsilon, r>0, m \in Z_{+}$such that

$$
E\left(G\left(\xi_{m}\right) \mid \xi_{1}=x\right) \leq G(x)-\epsilon
$$

for all $x$ satisfying the condition

$$
\max \left(x_{1}, x_{2}\right)>r
$$


Proof is analogous to the proof of lemma 3.3.

It follows from lemmas 3.3 and 3.4 that $G(x)$ is a Lyapounov function.

Second construction. This construction gives rise to a one-step Lyapounov function, i.e. $m=1$ in formula (3.1). To do this let us define the vectors

$$
\begin{gathered}
d_{0}=w\left(\lambda_{1} e_{1}(1)+\lambda_{2} e_{2}(2)\right), \\
d_{1}(1)=w \mu_{1}(1)\left(-e_{1}(1)+e_{2}(1)\right), \\
d_{1}(2)=-w \mu_{1}(2) e_{1}(2), \\
d_{2}(1)=-w \mu_{2}(1) e_{2}(1), \\
d_{2}(2)=w \mu_{2}(2)\left(-e_{2}(2)+e_{1}(2)\right) .
\end{gathered}
$$

It is easy to see that

$$
d_{0}+\sum_{i=1}^{2} \sum_{k=1}^{2} \rho_{i}(k) d_{i}(k)=0 .
$$

Let us introduce the vectors

$$
\begin{gathered}
M\left(k_{1}, k_{2}\right)=d_{0}+d_{1}\left(k_{1}\right)+d_{2}\left(k_{2}\right), \\
M_{1}\left(k_{1}\right)=d_{0}+d_{1}\left(k_{1}\right), \\
M_{2}\left(k_{2}\right)=d_{0}+d_{2}\left(k_{2}\right),
\end{gathered}
$$

where $k_{1}, k_{2}=1,2$.

The one-step mean jump vectors of $\xi_{n}$ are equal to:

$$
E\left(\xi_{n+1}-\xi_{n} \mid \eta_{n}=\alpha\right)= \begin{cases}M\left(k_{1}, k_{2}\right), & \text { if both nodes are busy, } \\ M\left(k_{1}\right), & \text { if node } 2 \text { is empty, } \\ M\left(k_{2}\right), & \text { if node } 1 \text { is empty }\end{cases}
$$

where $\alpha \in \mathcal{A}_{1} \times \mathcal{A}_{2}$ and $k_{i}$ are the types of calls being in progress at a given moment. Thus, the one-step mean jump vectors depend only on types of calls which are served at a given moment and do not depend on a service discipline.

Now we construct the first vector field in $R_{+}^{4}[6]$, which is multivalued in this case. The set of vectors

$$
N(x)=\left\{M\left(k_{1}, k_{2}\right), k_{1}, k_{2}=1,2: x_{1}\left(k_{1}\right), x_{2}\left(k_{2}\right)>0\right\}
$$


corresponds to the point $x \notin \Lambda_{1}^{(2)} \cup \Lambda_{2}^{(2)}$ (i.e., both nodes are busy), the set

$$
N_{1}(x)=\left\{M_{1}(k), k=1,2: x_{1}(k)>0\right\}
$$

corresponds to the point $x \in \Lambda_{1}^{(2)}$ (i.e, the second node is empty), the set

$$
N_{2}(x)=\left\{M_{2}(k), k=1,2: x_{2}(k)>0\right\}
$$

corresponds to the point $x \in \Lambda_{2}^{(2)}$ (i.e., the first node is empty). Here the vectors $M\left(k_{1}, k_{2}\right), M_{1}\left(k_{1}\right), M_{2}\left(k_{2}\right)$ are defined by $(3.8)$.

Consider level surfaces of the function $G(x)$. These surfaces are piecewise linear surfaces as by the construction $G(x)$ is a piecewise linear function. Consider the surface of level 1:

$$
S=\{x: G(x)=1\} .
$$

Then $S=S_{1} \cup S_{2}$, where

$$
\begin{aligned}
& S_{1}=\left\{G_{1}(x)=1, G_{2}(x) \leq 1\right\} \\
& S_{2}=\left\{G_{2}(x)=1, G_{1}(x) \leq 1\right\} .
\end{aligned}
$$

Take the hyperplanes

$$
P_{1}: G_{1}(x)=1, P_{2}: G_{2}(x)=1
$$

and corresponding the half-spaces

$$
P_{1}^{+}=G_{1}(x) \leq 1, P_{2}^{+}=G_{2}(x) \leq 1 .
$$

It is easy to see that $S$ is the surface of the two-face corner $P_{1}^{+} \cap P_{2}^{+}$in $R_{+}^{4}$ and $S_{1}, S_{2}$ are the faces of this corner.

It is convenient to reformulate lemmas 3.1 and 3.2 as follows.

Lemma 3.5 Any vector of sets $N(x), N_{1}(x)$ with an initial point on $P_{1}$ lies in $P_{1}^{+}$and any vector of sets $N(x), N_{2}(x)$ with an initial paint on $P_{2}$ lies int $P_{2}^{+}$.

Lemma 3.6 The face $S_{1}$ does not intersect the coordinate face $\Lambda_{2}^{(2)}$ and the face $S_{2}$ does not intersect the coordinate face $\Lambda_{1}^{(2)}$. 
Now we use smoothing to construct a smooth surface $S(\epsilon)$ which is an approximation for the piecewise linear surface $S$.

Lemma 3.7 For any $\epsilon>0$ there exists a smooth convex hypersurface $S(\epsilon)$ which satisfies the following conditions: for any $x \in S(\epsilon)$ holds

$$
\varrho(x, S)<\epsilon
$$

for any $x \in S$ does

$$
\varrho(x, S(\epsilon))<\epsilon,
$$

where $\varrho(x, y)$ is Euclidean metrics.

Proof. One can find a linear transformation such that the faces $S_{1}, S_{2}$ are orthogonal in a new basis. Therefore it is enough to consider the case when the faces are orthogonal. We use cylindrical surface $S(\epsilon)$ to smooth the surface $S$ in some neighbourhood of the intersection $S_{0}=S_{1} \cap S_{2}$. Let $S_{0}(\epsilon)$ be the intersection of $S(\epsilon)$ with this neighbourhood. Prolongating $S_{0}(\epsilon)$ by linearity we get the smooth convex hypersurface $S(\epsilon)$ which satisfies the conditions of the lemma.

Let us define the Lyapounov function

$$
\bar{G}(x)=a, \text { where } x \in a S(\epsilon), a>1 .
$$

The function $\bar{G}(x)$ is smooth by the construction.

By lemmas 3.5, 3.6 and 3.7 there exists $\sigma>0$ such that for any vector $\vec{v}(x)$ of the set $N(x), x \notin \Lambda_{1}^{(2)} \cup \Lambda_{2}^{(2)}$, or the set $N_{i}(x), x \in \Lambda_{i}^{(2)}, i=1,2$ the inequality

$$
(\nabla \bar{G}(x), \vec{v}(x))<-\sigma, \sigma>0
$$

is fulfilled uniformly over $x \in R_{+}^{4}$.

Using the principle of almost linearity [9] we obtain that $\bar{G}(x)$ is a Lyapounov function with $m=1$.

To prove the exponential estimates (2.2) and (2.3) we need some results from [8]. Let us consider a Markov chain with a countable state space $S$ and with transition probabilities $p_{i j}$. We suppose that the following conditions are fulfilled: there exist a non-negative function $f_{i}$ on $S$ and a positive integer $m$ such that 
i). the series

$$
\sum_{i} \exp \left(-b f_{i}\right)
$$

converges for any $b>0$;

ii). there exists $d>0$ such that $p_{i j}=0$ when $\left|f_{i}-f_{j}\right|>d$.

iii). there is $\epsilon>0$ such that

$$
\sum_{j} f_{j} p_{i j}^{(m)}-f_{i}<-\epsilon
$$

for all $i \in S \backslash B$, where $B$ is a finite subset of $S$ and $p_{i j}^{(m)}$ are the $m$-step transition probabilities.

If the above conditions are fulfilled then the following exponential estimates take place:

1. The exponential estimate for the stationary probabilities:

$$
\pi_{i}<C \exp \left(-\delta f_{i}\right)
$$

for some constants $C, \delta>0$.

2. Exponentially fast convergence to the stationary probabilities: there exist constants $C_{1}, \sigma>0$ such that

$$
\sum_{j}\left|p_{i j}^{(n)}-\pi_{j}\right|<C_{1} \exp (-\sigma n)
$$

for all $i \in S$ and for all $n>\sigma f_{i}$.

To prove the estimates $(2.2),(2.3)$ it is sufficient to verify the conditions i)-iii). Condition iii) has already been verified. Condition ii) follows from boundedness of jumps of $\xi_{n}$. Condition i) holds as our Lyapounov function is piecewise linear.

\section{Proof of theorem 2.}

In the case of disciplines with absolute priorities the communication network under study is represented by a random walk in $Z_{+}^{4}$. Ergodic properties of a general random walk in $Z_{+}^{4}$ have been studied in [5]. The methods suggested in this paper will be applied to analysis of our particular case. Now we recall some definitions from [5]. Let us consider a discrete time homogeneous 
Markov chain. The set of states is $Z_{+}^{4}$. Let $p_{x y}^{(m)}$ be the $n$-step transition probabilities,

$$
M(x)=\left(M_{1}(x), \ldots, M_{4}(x)\right)
$$

be the vector of mean jump from the point $x$ in one step. For any $\Lambda \subseteq$ $\{1, \ldots, 4\}$ the face $B(\Lambda)$ of $R_{+}^{4}$ is given by

$$
B(\Lambda)=\left\{\left(r_{1}, \ldots, r_{4}\right): r_{i}>0, i \in \Lambda, r_{i}=0, i \notin \Lambda\right\} .
$$

For short we shall write $\Lambda$ instead of $B(\Lambda)$.

A random walk which represents the network satisfies the following conditions.

Boundedness of jumps:

$$
p_{x y}=0, \text { for }\|x-y\|>1,
$$

where $\|x\|=\max _{i}\left|x_{i}\right|$.

Space homogeneity: for any $\Lambda$ and for any $a \in \Lambda \cap Z_{+}^{4}$

$$
p_{x y}=p_{x+a, y+a}
$$

for all $x \in \Lambda \cap Z_{+}^{4}, y \in Z_{+}^{4}$.

We define the first vector field to be constant on any $\Lambda$ :

$$
M_{\Lambda} \equiv M(x), x \in \Lambda \text {. }
$$

Let us define the induced chain. For any $\Lambda$ we choose an arbitrary point $a \in \Lambda \cap Z_{+}^{4}$ and draw a plane $C^{\Lambda}$ of dimension $4-|\Lambda|$ which is perpendicular to $\Lambda$ and contains point $a$. We define the induced chain with the state space $C^{\Lambda}$ and transition probabilities

$$
\wedge p_{x y}=p_{x y}+\sum_{z \neq y} p_{x z}, x, y \in C^{\Lambda},
$$

where the summation is performed over all $z \in Z_{+}^{4}$ such that the straight line connecting $z$ and $y$ is perpendicular to $C^{\Lambda}$.

We call the face $\Lambda$ ergodic if the induced chain is ergodic. For an ergodic induced chain let $\pi^{\Lambda}(z)$ be the stationary probabilities. We introduce the induced vector $V^{\Lambda}$ by setting

$$
V_{i}^{\Lambda}=0, i \notin \Lambda,
$$




$$
V_{i}^{\Lambda}=\sum_{z \in C^{\wedge}} \pi^{\wedge}(z) M_{i}(z), i \in \Lambda
$$

For $\Lambda=\{1, \ldots, 4\}$ we call $\Lambda$ ergodic and put

$$
V^{\Lambda}=M(x), x \in \Lambda \cap Z_{+}^{4} .
$$

Now we construct the second vector field for the random walk which represents the network under study. Let us consider the three-dimensional faces

$$
\begin{aligned}
& \Lambda_{1}^{(3)}=\left\{x_{2}(1)=0, x_{1}(1)>0, x_{1}(2)>0, x_{2}(2)>0\right\} \\
& \Lambda_{2}^{(3)}=\left\{x_{1}(2)=0, x_{1}(1)>0, x_{2}(1)>0, x_{2}(2)>0\right\} .
\end{aligned}
$$

The two-dimensional face

$$
\Lambda_{12}^{(2)}=\left\{x_{1}(2)=0, x_{2}(1)=0, x_{1}(1)>0, x_{2}(2)>0\right\}_{1}
$$

is the intersection of faces $\Lambda_{1}^{(3)}$ and $\Lambda_{2}^{(3)}$. The vectors of the first vector field on faces $\Lambda_{1}^{(3)}, \Lambda_{2}^{(3)}$ are equal to

$$
\begin{gathered}
M_{\Lambda_{1}^{(3)}} \equiv M_{1}^{(3)}=d_{0}+d_{1}(2)+d_{2}(2)= \\
=\left(\lambda_{2}-\mu_{2}(2)\right) e_{2}(2)+\lambda_{1} e_{1}(1)+\left(\mu_{2}(2)-\mu_{1}(2)\right) e_{1}(2) \\
M_{\Lambda_{2}^{(3)}} \equiv M_{2}^{(3)}=d_{0}+d_{1}(1)+d_{2}(1) \\
=\left(\lambda_{1}-\mu_{1}(1)\right) e_{1}(1)+\lambda_{2} e_{2}(2)+\left(\mu_{1}(1)-\mu_{2}(1)\right) e_{2}(1) .
\end{gathered}
$$

Observe that the condition $\rho_{1}(2)+\rho_{2}(1)>1$ implies

$$
\mu_{1}(1)>\mu_{2}(1), \mu_{2}(2)>\mu_{1}(2) \text {. }
$$

The vectors $M_{1}^{(3)}$ and $M_{2}^{(3)}$ lie on faces $\Lambda_{1}^{(3)}$ and $\Lambda_{2}^{(3)}$, respectively. It means that the union of faces $\Lambda_{1}^{(3)}$ and $\Lambda_{2}^{(3)}$ is the set of essential states of the random walk. So it is sufficient to consider the random walk on these faces.

As faces $\Lambda_{1}^{(3)}$ and $\Lambda_{2}^{(3)}$ form the set of essential states these faces are ergodic and the second vector field coincides with the first vector field:

$$
V_{\Lambda_{1}^{(3)}} \equiv V_{1}^{(3)}=M_{1}^{(3)}
$$




$$
V_{\Lambda_{2}^{(3)}} \equiv V_{2}^{(3)}=M_{2}^{(3)} \text {. }
$$

Consider the two-dimensional faces $\Lambda_{i}^{(2)}, i=1,2$, which are defined by (3.6). The vectors of the first vector field on these faces are

$$
\begin{aligned}
& M_{\Lambda_{1}^{(2)}} \equiv M_{1}^{(2)}=\lambda_{1} e_{1}(1)+\lambda_{2} e_{2}(2)-\mu_{1}(2) e_{1}(2), \\
& M_{\Lambda_{2}^{(2)}} \equiv M_{2}^{(2)}=\lambda_{1} e_{1}(1)+\lambda_{2} e_{2}(2)-\mu_{2}(1) e_{2}(1) .
\end{aligned}
$$

Lemma 4.1 Faces $\Lambda_{1}^{(2)}, \Lambda_{2}^{(2)}$ are ergodic and the vectors of the second vector field on these faces are

$$
\begin{aligned}
& V_{\Lambda_{1}^{(2)}} \equiv V_{1}^{(2)}=\lambda_{1} e_{1}(1)+\left(\lambda_{2}-\mu_{1}(2)\right) e_{1}(2), \\
& V_{\Lambda_{2}^{(2)}} \equiv V_{2}^{(2)}=\lambda_{2} e_{2}(2)+\left(\lambda_{1}-\mu_{2}(1)\right) e_{2}(1) .
\end{aligned}
$$

Proof. Consider the face $\Lambda_{2}^{(2)}$. For this face the induced chain is the simplest one-dimensional random walk which jumps on 1 with probability $c \lambda_{1}$ and on -1 with probability $c \mu_{1}(1)$, where $c=\left(\lambda_{1}+\mu_{1}(1)\right)^{-1}$. The stationary probability that such random walk is not in state 0 is equal to

$$
\frac{\lambda_{1}}{\mu_{1}(1)}=\rho_{1}(1) \text {. }
$$

So in accordance with (4.1) we have

$$
V_{2}^{(2)}=\rho_{1}(1) \operatorname{Pr}_{\Lambda_{2}^{(2)}} M_{2}^{(3)}+\left(1-\rho_{1}(1)\right) \operatorname{Pr}_{\Lambda_{2}^{(2)}} M_{2}^{(2)}
$$

Substituting the expressions for the vectors $M_{2}^{(3)}, M_{2}^{(2)}$ and taking their projections on the face $\Lambda_{2}^{(2)}$ we get

$$
V_{2}^{(2)}=\lambda_{2} e_{2}(2)+\left(\lambda_{1}-\mu_{2}(1)\right) e_{2}(1) .
$$

The vector $V_{1}^{(2)}$ is computed in the same way.

Remark that as only one component of each vector $M_{i}^{(3)}$ is negative there are not other two-dimensional ergodic faces and there are not one-dimensional faces. 
We define the second vector field $V(x)$ to be constant on any ergodic face $\Lambda$

$$
V(x) \equiv V^{\Lambda}, x \in \Lambda \text {. }
$$

Now we define the second vector field on non-ergodic faces. It can be multivalued. We say that $\Lambda$ is an outgoing face for $\Lambda_{1}, \Lambda_{1} \subset \Lambda$, if all coordinates $V_{i}^{\Lambda}$ for $i \in \Lambda \backslash \Lambda_{1}$ are positive. If $x \in \Lambda_{1}$, where $\Lambda_{1}$ is non-ergodic, then $V(x)$ takes all values $V_{\Lambda}$ for which $\Lambda$ is an outgoing face. For example, two vectors $V_{1}^{(3)}, V_{2}^{(3)}$ correspond to the face $\Lambda_{12}^{(2)}$. Further, the second vector field will be denoted by $\bar{V}$.

\section{Lemma 4.2 If}

$$
\rho_{1}<1, \rho_{2}<1, \text { and } \rho_{1}(2)+\rho_{2}(1)>1
$$

we never reach 0 moving along any trajectory of field $\bar{V}$.

Proof. Let us start at a point $x \in \Lambda_{1}^{(3)} \cup \Lambda_{2}^{(3)}$. Moving along a trajectory of field $\bar{V}$ we certainly reach one of the one-dimensional faces $\Lambda_{1}^{(1)}$ or $\Lambda_{2}^{(1)}$, where

$$
\begin{aligned}
& \Lambda_{1}^{(1)}=\left\{x_{1}(1)>0, \text { other coordinates }=0\right\}, \\
& \Lambda_{2}^{(1)}=\left\{x_{2}(2)>0, \text { other coordinates }=0\right\} .
\end{aligned}
$$

So one can suppose that a starting point lies on faces $\Lambda_{1}^{(1)}, \Lambda_{2}^{(1)}$, for example, $x \in \Lambda_{2}^{(1)}$. On this face the vector of the second field is $V_{2}^{(3)}$ which lies on the three-dimensional face $\Lambda_{2}^{(3)}$. So we shall move along the vector $V_{2}^{(3)}$ until we reach the two-dimensional face $\Lambda_{2}^{(2)}$. Further, we shall move along the vector $V_{2}^{(2)}$ on the face $\Lambda_{2}^{(2)}$ and reach a point $y$ lieing on the one-dimensional face $\Lambda_{1}^{(1)}$. It is easy to compute that

$$
y=\frac{\lambda_{2} x}{\mu_{2}(1)-\lambda_{1}} .
$$

The second vector field on the face $\Lambda_{1}^{(1)}$ is equal to $V_{1}^{(3)}$ where $V_{1}^{(3)}$ lies on the three-dimensional face $\Lambda_{1}^{(3)}$. Therefore, we shall move along the vector $V_{1}^{(3)}$ until reach the two-dimensional face $\Lambda_{1}^{(2)}$ and then moving along the vector $V_{1}^{(2)}$ on this face we reach a point $\tilde{x}$ belonging to the face $\Lambda_{2}^{(1)}$. Thus, the 
cycle has been finished. We have again reached the face $\Lambda_{2}^{(1)}$ we have started. It is easy to compute

$$
\begin{gathered}
\tilde{x}=\frac{\lambda_{1} y}{\mu_{1}(2)-\lambda_{2}}= \\
=\frac{\lambda_{1} \lambda_{2} x}{\left(\mu_{2}(1)-\lambda_{1}\right)\left(\mu_{1}(2)-\lambda_{2}\right)}
\end{gathered}
$$

The inequality

$$
\frac{\lambda_{1} \lambda_{2}}{\left(\mu_{2}(1)-\lambda_{1}\right)\left(\mu_{1}(2)-\lambda_{2}\right)}>1
$$

is equivalent to the inequality

$$
\rho_{1}(2)+\rho_{2}(1)>1
$$

Therefore, we never reach 0 moving along trajectories of field $\bar{V}$.

To prove that the network is transient we take the Lyapounov function $F(x)$ to be equal to the reaching time of state 0 starting at $x$ and moving along trajectories of field $\bar{V}$ in reverse direction. It is clear that for some $\delta>0$

$$
F(x+V(x))-F(x)>\delta,
$$

where $V(x)$ is a vector of field $\bar{V}$ in the point $x$. Then from the theorem 2.1 [8] it follows that the random walk is transient.

\section{$5 \quad$ Proof of Theorem 3.}

We prove this theorem for the case of the service discipline $D_{3}(p)$.

Let us define the linear functions

$$
\begin{gathered}
\hat{A}_{i}(x)=\frac{A_{i}(x)}{\rho_{i}}, i=1,2, \\
\hat{A}_{1,1}(x)=\frac{A_{1,1}(x)}{a_{1}}, \\
\hat{A}_{2,2}(x)=\frac{A_{2,2}(x)}{a_{2}}
\end{gathered}
$$


where the functions $A_{1,1}(x), A_{2,2}(x)$ are defined by (3.2), the function $A_{i}(x)$ is defined by (3.3), $\rho_{i}$ is the total load in the $i$-th node and constants $a_{i}$ are chosen in the special manner.

Let us define the Lyapounov function

$$
\dot{H}(x)=\max \left(\hat{A}_{1}(x), \hat{A}_{2}(x), \hat{A}_{1,1}(x), \hat{A}_{2,2}(x)\right) .
$$

We show that there exist $\epsilon>0$ and $m \in Z_{+}$such that

$$
E\left(H\left(\xi_{m}\right) \mid \xi_{1}=x\right) \leq H(x)-\epsilon
$$

for all $x \in Z_{+}^{4} \backslash B$, where $B$ is a finite set.

The proof of this statement is based on the following lemmas.

Lemma 5.1 The one-step mean increments of the functions $A_{i}\left(\xi_{n}\right), A_{1,1}\left(\xi_{n}\right)$, $A_{2,2}\left(\xi_{n}\right)$ are

$$
\begin{gathered}
E\left(A_{i}\left(\xi_{n+1}\right)-A_{i}\left(\xi_{n}\right) \mid \xi_{n, i}>0\right)=-1+\rho_{i}, \\
E\left(A_{1,1}\left(\xi_{n+1}\right)-A_{1,1}\left(\xi_{n}\right) \mid \xi_{n, 1}(1)>0\right)=-1+\rho_{1}(1), \\
E\left(A_{2,2}\left(\xi_{n+1}\right)-A_{2,2}\left(\xi_{n}\right) \mid \xi_{n, 2}(2)>0\right)=-1+\rho_{2}(2),
\end{gathered}
$$

Proof : analogous to the proof of lemma 3.1. It is based on explicit calculations.

Lemma 5.2 There exist constants $a_{1}$ and $a_{2}$ such that for $x \in \Lambda_{2}^{(2)}$

$$
\max \left(\hat{A}_{1}(x), \hat{A}_{1,1}(x)\right)<\max \left(\hat{A}_{2}(x), \hat{A}_{2,2}(x)\right),
$$

but for $x \in \Lambda_{1}^{(2)}$

$$
\max \left(\hat{A}_{1}(x), \hat{A}_{1,1}(x)\right)>\max \left(\hat{A}_{2}(x), \hat{A}_{2,2}(x)\right),
$$

Proof: similar to the proof of lemma 3.2.

Lemma 5.3 There exist such $\epsilon, r>0$ and $m \in Z_{+}$that

$$
E\left(H\left(\xi_{m}\right) \mid \xi_{1}=x\right) \leq H(x)-\epsilon
$$

for all $x$ satisfying the condition

$$
\min \left(x_{1}, x_{2}\right)>r
$$


Proof: it mimics the proof of lemma 3.3.

If $x$ is close by one of the two-dimensional faces $\Lambda_{1}^{(2)}, \Lambda_{2}^{(2)}$ the following result takes place.

Lemma 5.4 There exist $\epsilon, r>0$ and $m \in Z_{+}$such that

$$
E\left(H\left(\xi_{m}\right) \mid \xi_{1}=x\right) \leq H(x)-\epsilon
$$

for all $x$ satisfying the condition

$$
\max \left(x_{1}, x_{2}\right)>r
$$

Proof is analogous to the proof of lemma 3.3.

Remark. One can construct an one-step Lyapounov function, i.e. the inequality (5.1) is fulfilled for $m=1$, in the same way as it has been done in theorem 1.

The exponential estimates of the form (2.2), (2.3) are deduced from existence of the Lyapounov function in finite steps in the same way as in theorem 1 .

The disciplines $D_{1}(p), D_{2}(p)$ are considered by analogy with the above case. Moreover, it is known a solution of this task for general networks with the help of explicit formulae [10]. 


\section{References}

[1] Malyshev V.A. Random walks. The Wiener-Hopf equations in a quadrant of the plane. Galois automorphisms. Moscow State University Press. Moscow, 1970.

[2] Kelly F.P. Reversibility stochastic networks. Wiley, N.Y., 1979.

[3] Fayolle G., Malyshev V.A., Mensikov M.V., Sidorenko A.F. Lyapounov functions for Jackson networks. Rapport de Recherche, INRIA, 1991, no.13S0. To appear in Mathematics of Operations Research.

[4] Fayolle G., Ignatyuk I.A., Malyshev V.A., Mensikov M.V. Random walks in two dimensional complexes 1991. Queueing Systems,9(3):p.269$300,1991$.

[5] Ignatyuk I.A., Malyshev V.A. Classification of random walks in $Z_{+}^{4}$. Rapport de Recherche, INRIA, 1991, no.1516.

[6] Malyshev V.A. Networks and dynamical systems. Rapport de Recherche, INRIA, 1991, no.1468.

[7] Rybko A.N., Stolyar A.L. On the problem of ergodicity of Markov processes corresponding to the message switching networks. Preprint, 1991.

[8] Malyshev V.A., Menshikov M.V. Ergodicity, continuity and analyticity of countable Markov chains. Trans. Moscow Math. Soc., 1979, v.39, p.348 .

[9] Malyshev V.A. Classification of two-dimensional random walks and almost linear semimartingales. Dokl. Akad. Nauk USSR, 1972, v.202, no.3, p.526-528.

[10] Rybko A.N. Carrying capacity of two classes of networks with message commutation. Probl. per. inf., 1982, v.18, no.1, p.94-108. 
ISSN 0249 - 6399 